\title{
Pseudotumor-like syndrome and cerebrospinal fluid leak in meningiomas involving the posterior third of the superior sagittal sinus: report of $\mathbf{4}$ cases
}

\author{
Ashish H. Shah, MD, Michael E. Ivan, MD, MBS, and Ricardo J. Komotar, MD \\ Department of Neurological Surgery, University of Miami, Florida
}

Meningiomas that partially or completely occlude the superior sagittal sinus may create a pseudotumor-like syndrome in certain patients. These patients may have impaired CSF absorption as a result of higher proximal venous pressure. Higher pressures after resection may encumber adequate wound healing and worsen symptoms. Here, the authors present a small series of patients with meningiomas involving the posterior third of the superior sagittal sinus, with documented high intracranial pressure prior to surgery. This paper aims to address the proposed etiology of high intracranial pressure in these patients and its associated complications, including CSF leak, wound dehiscence, pressure-related headaches, and visual complaints. In this paper, the authors propose a management plan to avoid wound complications and pseudotumor-related complications. When considering surgical intervention for patients with compromise of the posterior third of the superior sagittal sinus, careful attention must be paid to addressing potentially elevated intracranial pressure perioperatively.

http://thejns.org/doi/abs/10.3171/2015.7.JNS15770

KEY WORDS pseudotumor; meningioma; superior sagittal sinus; complications; oncology

$\mathrm{P}$ ARASAGITTAL meningiomas that invade the superior sagittal sinus (SSS) have traditionally been difficult to manage. ${ }^{5,10}$ Adequate resection with or without adjuvant radiotherapy, depending on histology and extent of resection, may be the mainstay of treatment of symptomatic or growing lesions. However, SSS invasion may complicate surgical removal. As described by Sindou and Alvernia, meningiomas can be graded based on the degree of sinus involvement from I to VI, with Grade VI invasion indicating total invasion of the SSS with no free walls. ${ }^{1,12}$ In cases of near to total occlusion of the SSS, venous congestion may preclude CSF resorption. These meningiomas may partially or completely occlude the SSS, thereby increasing proximal venous pressure and decreasing CSF resorption.

We propose that parasagittal meningiomas that invade the posterior third of the parasagittal sinus may be predisposed to develop a pseudotumor-like condition, which may predispose patients to complications associated with high intracranial pressure (ICP). These issues include CSF leak, wound dehiscence, and pseudotumor-related symptoms.
Here we present 3 cases in which large parasagittal meningiomas that invaded the SSS demonstrated complications associated with high ICP that was likely due to sinus obstruction. Additionally, we present a fourth case in which these complications were avoided by addressing high intracranial pressure in the perioperative setting.

\section{Case Reports Case 1}

This 43-year-old woman initially presented with spontaneous nontraumatic CSF rhinorrhea with mild headaches. An extensive workup revealed a parasagittal meningioma involving the SSS near the torcular herophili (Fig. 1). The patient underwent a primary CSF leak repair performed by the otolaryngology team; subsequently, the CSF leak recurred, and a lumbar puncture revealed increased ICP higher than $25 \mathrm{~cm} \mathrm{H}_{2} \mathrm{O}$. Surveillance of the meningioma revealed SSS occlusion at the posterior third. As a result, the patient was scheduled for ventriculoperitoneal shunt placement to help resolve the CSF leak and reduce ICP. The 

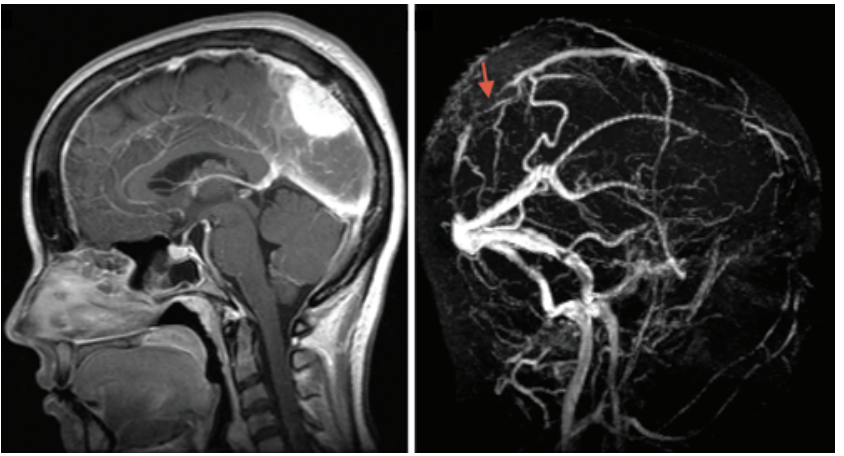

FIG. 1. Left: Preoperative postcontrast sagittal T1-weighted MR image of the brain demonstrating a posterior parasagittal meningioma. Right: Magnetic resonance venography demonstrating near-total occlusion of the SSS (arrow). Figure is available in color online only.

patient was then scheduled for resection of the torcular meningioma. The patient tolerated the procedure well without any deficits or complications. There were no issues with wound healing and no further CSF leaks were reported.

\section{Case 2}

This 62-year-old man with no significant medical history, presenting with an approximately 1-year history of headaches and visual disturbances in the inferior fields and MRI with a large occipital meningioma (Fig. 2). This tumor had significant invasion of the posterior aspect of the SSS. The patient underwent an occipital craniotomy for meningioma resection without any complications. Postoperatively, there was still evidence of stenosis of the SSS; however, the sinus still appeared patent. In a delayed fashion, the patient had evidence of a CSF leak from the wound. The patient was scheduled for a wound revision with placement of a lumbar drain. At that time, the opening pressure during the placement of this drain was elevated to higher than $20 \mathrm{~cm} \mathrm{H}_{2} \mathrm{O}$. With evidence of persistent elevated ICP, the lumbar drain was converted to a lumboperitoneal shunt to ensure adequate wound healing and prevention of worsening symptomatology. Postoperatively, the wound healed
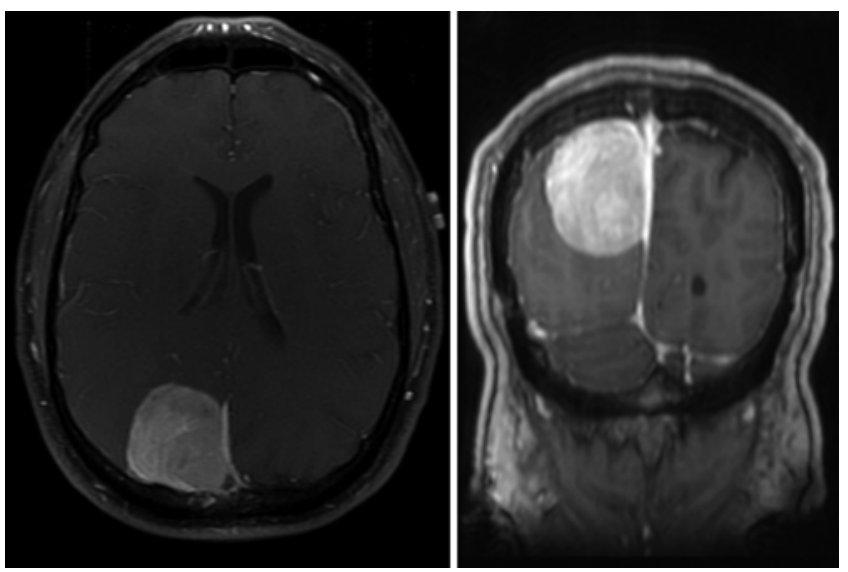

FIG. 2. Preoperative axial T1-weighted axial (left) and coronal (right) MR images of the brain with contrast demonstrating a large $4.5 \times 3.5-$ $\mathrm{cm}$ occipital meningioma bordering the SSS. well and the patient remained at his neurological baseline. Postoperatively, his visual fields improved.

\section{Case 3}

This 43-year-old otherwise healthy man noticed a severe sudden onset headache and left homonymous hemianopia 1 week prior to his visit. Imaging demonstrated a right occipital parafalcine meningioma. The posterior SSS in the region of the tumor appeared occluded (Fig. 3). A right occipital craniotomy for tumor resection was performed. Postoperatively, the SSS had residual stenosis but was patent, and the patient developed a wound dehiscence that necessitated wound revision. In the ensuing weeks, a CSF leak was noted at the wound site. A lumbar drain was placed with an opening pressure again noted to be higher than $20 \mathrm{~cm}$ of $\mathrm{H}_{2} \mathrm{O}$. The drain was continued and was safely discontinued once ICP was noted to be low. The patient was discharged home in a stable fashion with a well-healed wound. Follow-up visits by ophthalmology did not reveal any evidence of papilledema.

\section{Case 4}

This 46-year-old woman had a history of increasing headaches and numbness of her right leg over the last few weeks. The patient underwent MRI of the brain, which revealed a posterior left parietal meningioma with effacement of the SSS (Fig. 4). Preoperatively, a lumbar puncture revealed an opening pressure of $28 \mathrm{~cm} \mathrm{H}_{2} \mathrm{O}$; therefore, a lumbar drain was placed. A subtotal resection was achieved, and the lumbar drain was maintained for 5 days postoperatively. Postoperative MRI demonstrated residual stenosis of the posterior third of the SSS; however, the sinus appeared still patent. After 5 days, there were no signs of increased ICP or CSF leakage from the wound. The drain was discontinued, and the patient was discharged home without any complications.

\section{Discussion}

Meningiomas that invade the posterior SSS may produce a pseudotumor-like condition that predisposes patients to symptoms of increased ICP. Idiopathic intracranial hypertension (pseudotumor cerebri) is a complicated entity that is clinically defined by the modified Dandy crite-
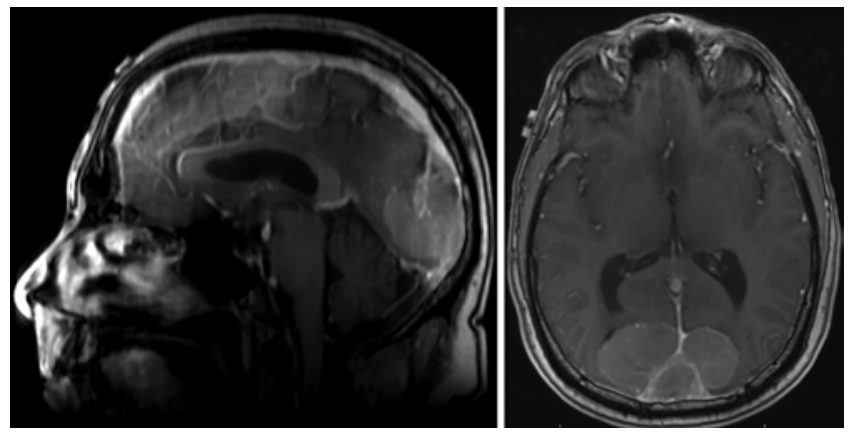

FIG. 3. Preoperative sagittal T1-weighted (left) and axial (right) MR images of the brain with contrast demonstrating a large dural-based lesion encompassing the SSS. 


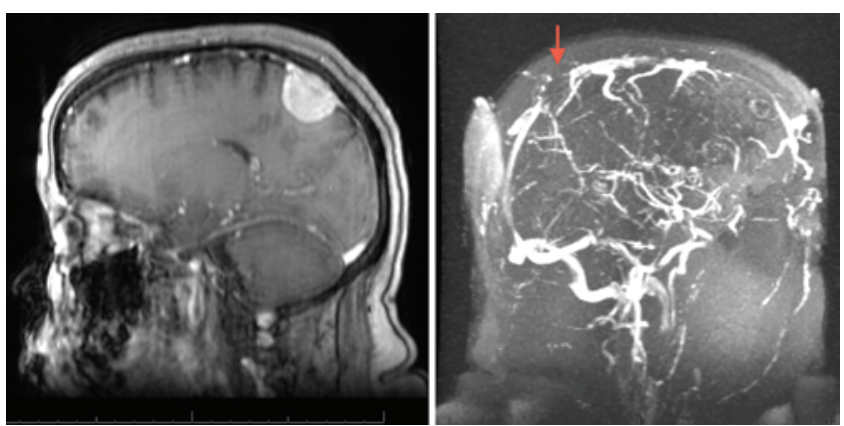

FIG. 4. Preoperative T1-sagittal MR image of the brain with contrast (left) showing a parietal 2.4-cm meningioma with occlusion/stenosis of the SSS on MRV (right; arrow). Figure is available in color online only.

ria:7,11 1) signs/symptoms of increased ICP; 2) no localizing findings (not including sixth cranial nerve palsy); 3 ) increased ICP; 4) no other identifiable causes of increased ICP; and 5) essentially unrevealing neuroimaging. Occlusion of the venous draining system can produce a pseudotumor-like picture (the Dandy criteria cannot be directly applied because of the neuroradiological criterion).

Cranial venous outflow abnormalities are estimated to be found in $20 \%$ of all pseudotumor patients. The most common site of abnormalities is the transverse sinus, followed by the SSS. ${ }^{8}$ Typically, obstruction of venous outflow is secondary to hematological pathology (hypercoagulable state), iatrogenic causes (embolization, surgery, or infection), or trauma (depressed skull fracture occluding sinus). ${ }^{3,6,9,13}$ A pseudotumor-like syndrome primarily due to tumor compression of the venous sinus drainage remains a rare, but known, etiology. Previously, intracranial hypertension had been well described due to jugular compression/occlusion secondary to glomus jugulare tumors. ${ }^{2,4}$ In these instances, resection of the lesion most often improved visual acuity and lowered ICP. Although rare, lesions such as skull fractures compressing the posterior third of the SSS can produce this pseudotumor-like syndrome. ${ }^{3,8}$ These patients typically presented with worsening vision and documented papilledema; however, we present a series of patients with a separate, but physiologically similar, clinical picture.

In our patient series of meningiomas compressing the posterior SSS, CSF leaks were noted, either spontaneously (Case 1) or postoperatively (Cases 2 and 3). For the latter 2 patients, intraoperative monitoring revealed elevated ICP without evidence of a CSF outflow obstruction or hydrocephalus. We hypothesize that these patients experienced a complex intracranial hypertension that was instigated by tumor-related venous obstruction and worsened by surgical proteinaceous products. A lumbar puncture was performed to confirm a suspicion of high ICP in the setting of venous obstruction.

\section{Mechanism of ICP}

Currently, the pathophysiology of benign intracranial hypertension or pseudotumor remains elusive. Most commonly, it is assumed that there is an inherent deficit in the absorption of CSF at the arachnoid villi. In the case of venous outflow obstruction, impaired CSF absorption may be secondary to an impaired pressure gradient between CSF outflow and venous pressure. If this gradient is abnormally low, CSF cannot escape into normal venous channels, thereby prompting intracranial hypertension. In all patients included in our study, there was clear evidence of venous compression/occlusion. Nevertheless, some of the patients in our study had confounding factors that may have further elevated the ICP such as large supratentorial lesions.

In the setting of increased ICP, appropriate wound healing may be difficult as CSF may escape through the galeal layers. One of the first signs of increased ICP may be a CSF leak in the setting of a pseudomeningocele at the surgical site. In 2 of our patients, increased ICP predisposed a bulging pseudomeningocele with CSF leak (Cases 2 and 3 ). Appropriate management of this condition may require either CSF diversion or reduction of the venous obstruction. Delayed wound healing and CSF leaks occurred in the latter 2 patients despite resection of the instigating tumor. In all of our patients, small residual tumor was left on the SSS to avoid any sinus injury. We hypothesize that the SSS remained occluded after surgery; therefore, the pseudotumor-like condition persisted. Evaluation of ICP during surgery of meningiomas involving the posterior third of the SSS may be useful in evaluating if that patient has intracranial hypertension and the need for permanent CSF diversion.

\section{Our Paradigm}

In patients with suspected increased ICP secondary to occlusion of the posterior third of the SSS, we would recommend the following. In symptomatic patients who require resection of the lesion, a preoperative assessment (funduscopy for papilledema) and lumbar drain to assess opening pressure, and provide brain relaxation may be helpful. If high opening pressure is documented, the lumbar drain can be maintained for 3-5 days postoperatively to allow for adequate wound healing. Performing a lumbar puncture in patients with pseudotumor-like conditions is mostly safe as there is no evidence of any obstruction; however, considerations must be taken to avoid large pressure changes in the intracranial compartment that would predispose patients to herniation. Therefore, a low-volume spinal tap $(<5 \mathrm{ml})$ may be safe in these patients with large meningiomas prior to resection. Postoperatively, a lumbar drain clamping trial in these patients would be necessary to assess for signs of increased ICP (CSF leak from wound site, CSF leak from clamped lumbar drain, blurry vision, intractable headaches, and documented high ICP). If the patient has no signs of increased ICP, the drain can be removed. In patients in whom a clamping trial fails, the lumbar drain may be extended for a few more days, but permanent CSF diversion may be necessary. Our flow diagram below outlines this decision-making paradigm (Fig. 5).

Further investigation into the best practice is warranted to prevent wound healing issues.

\section{Conclusions}

Occlusion of the posterior third of the SSS by tumors may theoretically predispose patients to a pseudotumorlike condition. The pathophysiology of this condition may 


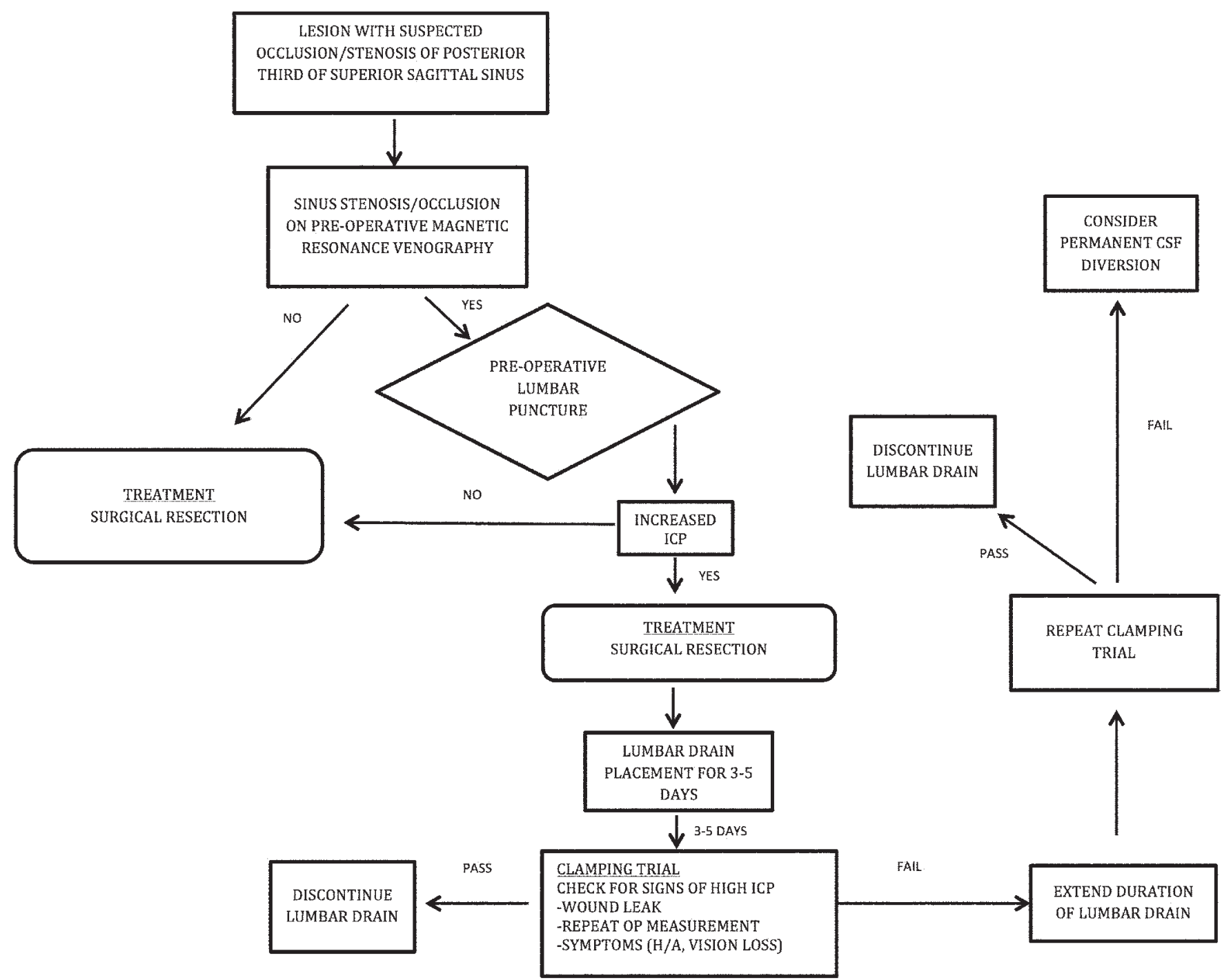

FIG. 5. Treatment paradigm.

be related to increased venous pressure reducing the CSF outflow gradient. Nevertheless, the mechanism of this increased ICP in the setting of a lesion producing sinus stenosis remains unclear. Removal of the instigating factor (tumor) may not always relieve increased ICP; therefore, CSF diversion may be necessary. Our series of patients all demonstrated CSF leaks in the setting of occlusion/compression of the posterior third of the SSS. These complications could be related to the presence of increased ICP in the setting of a pseudotumor-like condition. Early detection of this entity may prevent postoperative sequelae such as delayed wound healing, CSF leaks, or meningitis. Further evaluation into these related processes is needed develop a best practice for treatment.

\section{References}

1. Alvernia JE, Sindou MP: Preoperative neuroimaging findings as a predictor of the surgical plane of cleavage: prospective study of 100 consecutive cases of intracranial meningioma. J Neurosurg 100:422-430, 2004
2. Angeli SI, Sato Y, Gantz BJ: Glomus jugulare tumors masquerading as benign intracranial hypertension. Arch Otolaryngol Head Neck Surg 120:1277-1280, 1994

3. Bodkin PA, Hassan MF, Kane PJ, Brady N, Whittle IR: 'Surgical' causes of benign intracranial hypertension. J R Soc Med 101:259-261, 2008

4. Brookes GB, Graham MD: Benign intracranial hypertension complicating glomus jugulare tumor surgery. Am J Otol 5:350-354, 1984

5. DiMeco F, Li KW, Casali C, Ciceri E, Giombini S, Filippini $\mathrm{G}$, et al: Meningiomas invading the superior sagittal sinus: surgical experience in 108 cases. Neurosurgery 55:12631274, 2004

6. Fuentes S, Metellus P, Levrier O, Adetchessi T, Dufour H, Grisoli F: Depressed skull fracture overlying the superior sagittal sinus causing benign intracranial hypertension. Description of two cases and review of the literature. Br J Neurosurg 19:438-442, 2005

7. Johnston I, Hawke S, Halmagyi M, Teo C: The pseudotumor syndrome. Disorders of cerebrospinal fluid circulation causing intracranial hypertension without ventriculomegaly. Arch Neurol 48:740-747, 1991

8. Johnston I, Kollar C, Dunkley S, Assaad N, Parker G: 
Cranial venous outflow obstruction in the pseudotumour syndrome: incidence, nature and relevance. J Clin Neurosci 9:273-278, 2002

9. Kollar CD, Johnston IH: Pseudotumour after arteriovenous malformation embolisation. J Neurol Neurosurg Psychiatry 67:249, 1999 (Letter)

10. Nowak A, Dziedzic T, Czernicki T, Kunert P, Marchel A: Surgical treatment of parasagittal and falcine meningiomas invading the superior sagittal sinus. Neurol Neurochir Pol 48:174-180, 2014

11. Pillai A, Kumar S, Kumar A, Panikar D: An unusual parasagittal tumour with acute blindness and response to cerebrospinal fluid shunting. J Clin Neurosci 14:1112-1116, 2007

12. Sindou MP, Alvernia JE: Results of attempted radical tumor removal and venous repair in 100 consecutive meningiomas involving the major dural sinuses. J Neurosurg 105:514525,2006

13. van den Brink WA, Pieterman H, Avezaat CJ: Sagittal sinus occlusion, caused by an overlying depressed cranial fracture, presenting with late signs and symptoms of intracranial hypertension: case report. Neurosurgery 38:1044-1046, 1996

\section{Disclosures}

The authors report no conflict of interest concerning the materials or methods used in this study or the findings specified in this paper.

\section{Author Contributions}

Conception and design: all authors. Acquisition of data: all authors. Analysis and interpretation of data: all authors. Drafting the article: all authors. Critically revising the article: all authors. Reviewed submitted version of manuscript: all authors. Administrative/technical/material support: Komotar. Study supervision: Komotar, Ivan.

\section{Correspondence}

Ricardo J. Komotar, Department of Neurological Surgery, Surgical Neurooncology, University of Miami Hospital, University of Miami School of Medicine, 1095 NW 14th Terrace, Rm. 2-06, Miami, FL 33136. email: rkomotar@med.miami.edu. 